\title{
Analysis of the clinical effect of osimertinib on 90 cases with advanced lung adenocarcinoma
}

\author{
Zhiqiang Gao ${ }^{1 \#}$, Weimin Wang ${ }^{1 \#}$, Aiqin Gu${ }^{1}$, Jianhong Lu ${ }^{2}$, Aimi Huang ${ }^{1}$, Liwen Xiong ${ }^{1}$, Baohui Han ${ }^{1}$, \\ Liyan Jiang ${ }^{1}$, Chunlei Shi ${ }^{1}$ \\ ${ }^{1}$ Department of Respiratory Medicine, Shanghai Chest Hospital, Shanghai Jiao Tong University, Shanghai, China; ${ }^{2}$ International Medical Care \\ Center, Shanghai General Hospital, Shanghai Jiao Tong University School of Medicine, Shanghai, China \\ Contributions: (I) Conception and design: A Gu, J Lu; (II) Administrative support: B Han; (III) Provision of study materials or patients: A Gu, J Lu, \\ L Xiong, B Han, L Jiang, C Shi; (IV) Collection and assembly of data: Z Gao, W Wang, A Huang; (V) Data analysis and interpretation: Z Gao, W \\ Wang; (VI) Manuscript writing: All authors; (VII) Final approval of manuscript: All authors. \\ \#These authors contributed equally to this work and are co-first authors. \\ Correspondence to: Aiqin Gu. Department of Respiratory Medicine, Shanghai Chest Hospital, Shanghai Jiao Tong University, Shanghai, China. \\ Email: guaiqin11@126.com; Jianhong Lu. International Medical Care Center, Shanghai General Hospital, Shanghai Jiao Tong University School of \\ Medicine, Shanghai, China. Email: Lujhwin@163.com.
}

Background: Primary or secondary drug resistance of epidermal growth factor receptor-tyrosine kinase inhibitors (EGFR-TKI) is a new challenge in the treatment of advanced non-small cell lung cancer (NSCLC). Osimertinib is a third-generation EGFR-TKI, and its efficacy and safety in NSCLC patients with firstgeneration EGFR-TKI resistance, especially lung adenocarcinoma, are not yet clear. The purpose of this study was to observe the efficacy and adverse reactions of osimertinib in the treatment of patients with advanced lung adenocarcinoma.

Methods: From January 2017 to December 2018, 90 patients with advanced (stage IV) lung adenocarcinoma were diagnosed in Shanghai Chest Hospital. The disease of these patients (94.4\%) progressed after first-line EGFR-TKI treatment, and $43.3 \%$ of patients received third-line or beyond thirdline treatment. The efficacy and adverse reactions of osimertinib treatment were observed.

Results: Among 90 patients with advanced lung adenocarcinoma, 57 (63.3\%) achieved partial response (PR), 27 (30.0\%) had stable disease (SD), and 6 (6.7\%) had progressive disease (PD). The objective response rate (ORR) was $63.3 \%$, and the disease control rate (DCR) was $93.3 \%$. The median progression-free time (mPFS) was 10.41 months (95\% CI: 8.91-11.91 months), and the median overall survival (mOS) was 31.37 months (95\% CI: 26.37-36.37 months). The incidence of adverse events of degree 3 and above was $7.78 \%$. The main adverse events were diarrhea (28.9\%) and rash (24.4\%). After symptomatic treatment, the incidence of adverse events was significantly reduced.

Conclusions: Osimertinib has a definite curative effect in the treatment of patients with advanced lung adenocarcinoma, and the incidence of adverse reactions is low.

Keywords: Osimertinib; lung adenocarcinoma; efficacy; T790M mutation

Submitted Apr 21, 2020. Accepted for publication Jul 21, 2020.

doi: $10.21037 /$ tlcr-20-685

View this article at: http://dx.doi.org/10.21037/tlcr-20-685

\section{Introduction}

Non-small cell lung cancer (NSCLC) accounts for $80-85 \%$ of all lung cancer (1). The mutation rate of epidermal growth factor receptor (EGFR) in NSCLC patients in East Asia is the highest (40-55\%). EGFR tyrosine kinase inhibitors (EGFR-TKIs) such as gefitinib and erlotinib have achieved remarkable results in the treatment of advanced 
NSCLC mutated by EGFR. Now, the primary or secondary resistance of EGFR-TKIs in the treatment of NSCLC has become the new challenge in the treatment of advanced NSCLC.

Development of the T790M resistance mutation in exon 20 of the EGFR gene is the major mechanism of acquired resistance, accounting for approximately $60 \%$ of treatment failure after the use of first-generation and secondgeneration EGFR-TKIs. MET amplification is another important mechanism detected in approximately $5 \%$ to $22 \%$ of relapsed patients. In recognition of this challenge, thirdgeneration EGFR-TKIs, such as osimertinib (TAGRISSO or AZD9291), rociletinib (CO1686), olmutinib (HM61713), nazartinib (EGF816), naquotinib (ASP8273), mavelertinib (PF-0647775), and AC0010, have been developed that selectively and irreversibly inhibit EGFR carrying the common "sensitive" mutations, 19del and L858R, and the resistant T790M mutation while sparing wild-type (WT) EGFR.

Osimertinib is a third generation EGFR-TKI, which selectively acts on NSCLC patients with EGFR-TKI sensitive mutations and secondary T790M-resistant mutations (2). The efficacy and safety of osimertinib in the first generation of EGFR-TKI-resistant NSCLC patients are unclear.

In this study, 90 patients with EGFR gene mutationpositive advanced lung adenocarcinoma treated in Shanghai Chest Hospital from January 2017 to December 2018 were analyzed retrospectively.

We present the following article in accordance with the STROBE reporting checklist (available at http://dx.doi. org/10.21037/tlcr-20-685).

\section{Methods}

\section{Clinical data}

Research start and end time: January 2017 to December 2018.

Participants: ninety patients with advanced (stage IV) lung adenocarcinoma were diagnosed in Shanghai Chest Hospital. After the first-line EGFR-TKI treatment, 43.3\% (39/90) of the patients received third-line or beyond thirdline treatment. Among these participants, 15 patients underwent second biopsy, 55 patients underwent peripheral blood test for T790M gene mutation, and 64 patients were positive for T790M mutation after EGFR-TKI resistance.

Among them, 39 cases were male (43.3\%), and 51 cases were female $(56.7 \%)$. The age range was $28-$ 83 years (median age was 61 years), and the average age was 60.1 years. For staging, 90 cases were stage IV, and 20 of these were patients with postoperative recurrence and metastasis; 21 of patients had brain metastasis (23.3\%), 65 patients had less than 3 sites of metastasis $(72.2 \%)$, 11 patients were smokers $(12.2 \%)$, and 66 patients were nonsmokers $(73.3 \%)$. All of the above cases were proven to have adenocarcinoma of the lung by cytology or histology, and measurable lesions were found in the lung (see Table 1).

\section{Therapeutic methods}

The administration plan was oral osimertinib (80 mg) once a day until tumor progression or intolerable adverse reactions. The patient did not receive any other systemic antitumor therapy during the treatment with osimertinib.

\section{Curative effect evaluation}

We evaluated the curative effect according to the Response Criteria In Solid Tumors 1.1 (RECIST 1.1) using the following definitions: complete response (CR), partial response (PR), stable disease (SD), and disease progression (PD). The objective response rate (ORR) was defined as follows: $(\mathrm{ORR})=(\mathrm{CR}+\mathrm{PR}) /$ total cases $\times 100 \%$. The disease control rate (DCR) was defined as follows: (DCR) $=(\mathrm{CR}+\mathrm{PR}+\mathrm{SD}) /$ total cases $\times 100 \%$. Progression-free survival (PFS) was defined as the time from the beginning of treatment to the progress of disease or death caused by any reason. Overall survival (OS) was defined as the time from the beginning of treatment to the death of patients. Side effects were evaluated according to the National Cancer Institute general toxicity standard 4.0 (CTCAE v4.0).

Four weeks after the initial oral administration of osimertinib, the patients were reexamined with chest computed tomography (CT) to evaluate the therapeutic effect. Routine blood and liver and kidney function tests were conducted regularly during the administration period. Chest imaging examination was carried out regularly and outpatient follow-up was carried out on time. Patients who failed to receive outpatient service were followed up regularly by telephone.

The study was conducted in accordance with the Declaration of Helsinki (as revised in 2013). Because of the retrospective nature of the research, the requirement for ethical approval and informed consent was waived. 
Table 1 Clinical characteristics of 90 patients with EGFR mutation-positive advanced lung adenocarcinoma

\begin{tabular}{|c|c|c|}
\hline Characteristic & $\mathrm{N}$ & $\%$ \\
\hline \multicolumn{3}{|l|}{ Gender } \\
\hline Male & 39 & 43.3 \\
\hline Female & 51 & 56.7 \\
\hline \multicolumn{3}{|l|}{ Age } \\
\hline$<60$ & 42 & 46.7 \\
\hline$\geq 60$ & 48 & 53.3 \\
\hline \multicolumn{3}{|l|}{ Smoking history } \\
\hline Smoker & 11 & 12.2 \\
\hline Non-smoker & 66 & 73.3 \\
\hline Unknown & 13 & 14.5 \\
\hline Second biopsy & 15 & 16.7 \\
\hline \multicolumn{3}{|l|}{ EGFR initial status } \\
\hline $19 \mathrm{del}$ & 59 & 65.6 \\
\hline $21 L 858 R$ & 18 & 20.0 \\
\hline Unknown or rare mutation & 13 & 14.4 \\
\hline \multicolumn{3}{|l|}{ T790M status } \\
\hline$(+)$ & 64 & 71.1 \\
\hline$(-)$ & 6 & 6.7 \\
\hline Unknown & 20 & 22.2 \\
\hline \multicolumn{3}{|c|}{ Brain assessment before therapy } \\
\hline Brain metastasis $(+)$ & 21 & 23.3 \\
\hline Brain metastasis (-) & 69 & 76.7 \\
\hline \multicolumn{3}{|c|}{ Number of metastasis sites before therapy } \\
\hline$<3$ & 65 & 72.2 \\
\hline$\geq 3$ & 25 & 27.8 \\
\hline
\end{tabular}

+ , positive; -, negative. EGFR, epidermal growth factor receptor.

\section{Statistical analysis}

SPSS statistics version 22 software was used for statistical analysis. The survival analysis of PFS and OS was calculated by Kaplan-Meier method. The comparison between different groups was performed by log-rank test. A P value $<0.05$ was considered to indicate significant difference.

\section{Results}

\section{Clinical efficacy}

In the follow-up that lasted to January 2020, 57 patients (63.3\%) achieved PR, 27 patients (30.0\%) achieved SD, and 6 patients $(6.7 \%)$ achieved PD. The ORR was $63.3 \%$ (57/90), and the DCR was 93.3\% (84/90). Median PFS (mPFS) was 10.41 months (95\% CI, 8.91-11.91 months). The median OS (mOS) was 31.37 months (95\% CI, 26.3736.37 months).

In the subgroup analysis, the ORR of patients with the 19 del mutation before treatment was $71.2 \%$, and the DCR was $96.6 \%$, while the mPFS was 11.67 months, and the mOS was 34.67 months. The ORR of patients with the $21 \mathrm{~L} 858 \mathrm{R}$ mutation was $61.1 \%$, and the DCR was $94.4 \%$, while the mPFS was 9.57 months, and the mOS was 30.27 months. The ORR of patients with unknown and rare mutations was $30.8 \%$, and the DCR was $76.9 \%$, while the mPFS was 6.99 months, and the mOS was 31.37 months.

The ORR of patients with a positive T790M mutation was $70.3 \%$ while that of patients with a negative or undetected T790M mutation was $46.2 \%$. The difference was statistically significant $(\mathrm{P}=0.031)$. The $\mathrm{DCR}$ of patients with a positive T790M mutation was $93.8 \%$, and the DCR of patients with a negative or undetected T790M mutation was $92.3 \%(\mathrm{P}=0.828)$. The $\mathrm{mPFS}$ and $\mathrm{mOS}$ of patients with a positive T790M mutation were 12.30 and 37.27 months, respectively, while the $\mathrm{mPFS}$ and $\mathrm{mOS}$ of patients with a negative or undetected $T 790 M$ mutation were 6.57 and 25.17 months, respectively. The difference was statistically significant $(\mathrm{P}=0.004$ and $\mathrm{P}=0.021)$ (Figure 1).

Twenty-one patients with brain metastasis had an ORR of $71.4 \%$, a DCR of $95.2 \%$, an mPFS of 10.21 months, and an mOS of 29.57 months. Gender, age, smoking status, and number of transferred organs had no effect on ORR, DCR, PFS, and OS (see Tables 2,3).

\section{Safety analysis}

The incidence of adverse events of grade 3 and above was $7.78 \%$ (7/90). Two patients stopped taking osimertinib because of adverse events. The most common adverse events were diarrhea (28.9\%), skin rash (24.4\%), dry skin $(17.8 \%)$, and paronychia (13.3\%). The adverse events could be alleviated after symptomatic treatment (Table 4). 

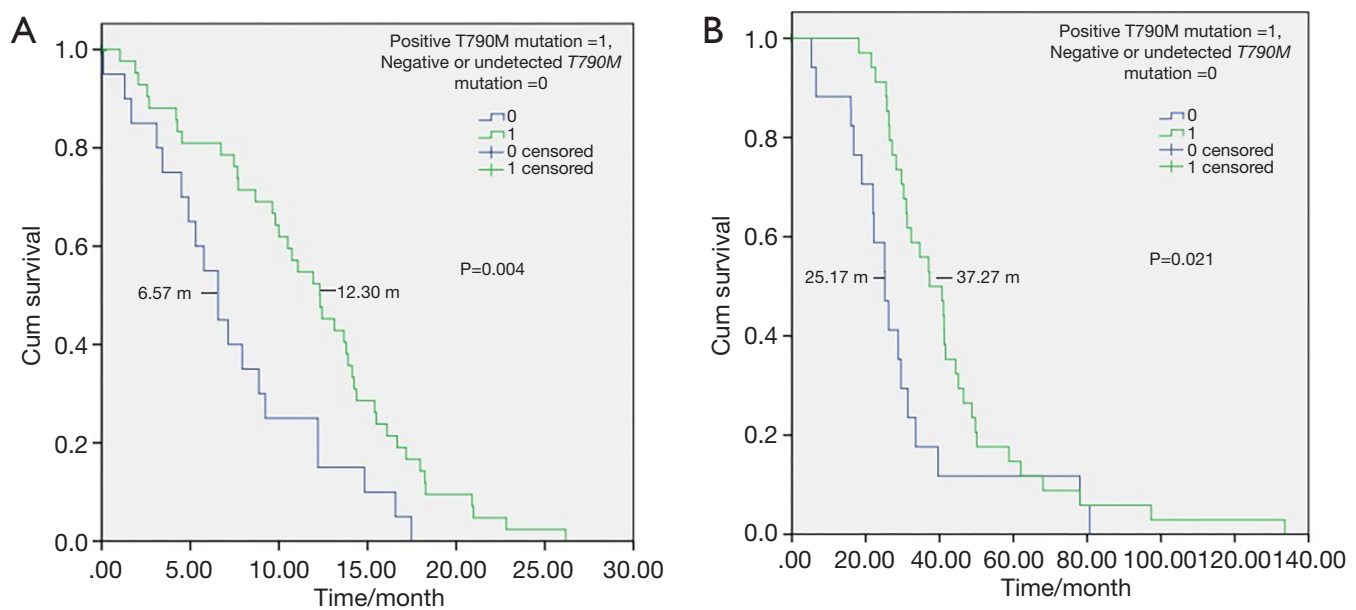

Figure 1 Progression-free survival and overall survival curves of patients with advanced lung adenocarcinoma treated with osimertinib. (A) Progression-free survival curves; (B) overall survival curves.

Table 2 Analysis of the response patients with advanced lung adenocarcinoma treated with osimertinib

\begin{tabular}{|c|c|c|c|c|c|}
\hline Characteristic & $\mathrm{N}$ & ORR (\%) & $\mathrm{P}$ & DCR (\%) & $\mathrm{P}$ \\
\hline Male & 39 & $26(66.7)$ & 0.566 & $34(87.2)$ & 0.105 \\
\hline Female & 51 & $31(60.8)$ & & $50(98.0)$ & \\
\hline \multicolumn{6}{|l|}{ Age } \\
\hline \multicolumn{6}{|l|}{ Smoking history } \\
\hline Smoker & 12 & $8(66.7)$ & 0.692 & $11(91.7)$ & 0.432 \\
\hline Non-smoker & 65 & $41(63.1)$ & & $62(95.4)$ & \\
\hline $21 L 858 R$ & 18 & $11(61.1)$ & & $17(94.4)$ & \\
\hline Unknown or rare mutation & 13 & $4(30.8)$ & & $10(76.9)$ & \\
\hline \multicolumn{6}{|l|}{ T790M status } \\
\hline$(+)$ & 64 & $45(70.3)$ & 0.031 & $60(93.8)$ & 0.828 \\
\hline$(-)$ or unknown & 26 & $12(46.2)$ & & $24(92.3)$ & \\
\hline \multicolumn{6}{|c|}{ Brain assessment before therapy } \\
\hline Brain metastasis $(+)$ & 21 & $15(71.4)$ & 0.379 & $20(95.2)$ & 0.92 \\
\hline Brain metastasis $(-)$ & 69 & $42(60.9)$ & & $64(92.8)$ & \\
\hline
\end{tabular}

+, positive; -, negative. ORR, objective response rate; DCR, disease control rate. 
Table 3 Survival analysis of patients with advanced lung adenocarcinoma treated with osimertinib

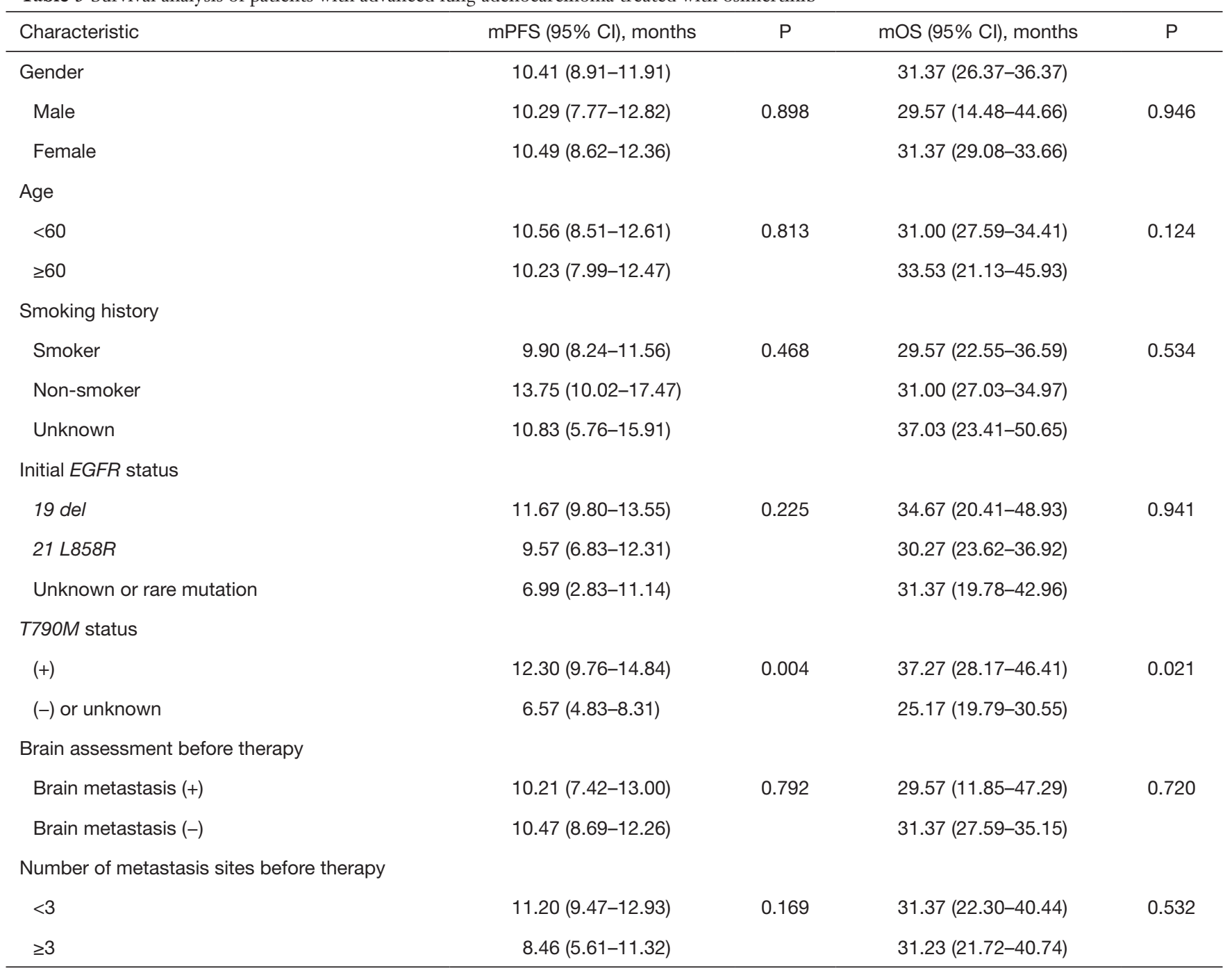

+, positive; -, negative. mPFS, median progression-free survival; mOS, median overall survival.

\section{Detection method for the T790M gene mutation}

The mutation of the T790M gene was detected in 70 patients with 11 biopsies, 4 biopsies and peripheral blood tests, and 55 peripheral blood tests. The detection methods included polymerase chain reaction (PCR), amplification refractory mutation system (ARMS), microdrop digital PCR (ddPCR), cobas, and next-generation sequencing (NGS) (Table 5).

\section{Discussion}

Acquisition of the EGFR T790M mutation is the most common resistance mechanism, accounting for $50-60 \%$ of progression after first-line EGFRTKIs. The presence of the
T790M variant reduces the ability of the reversible EGFRTKIs, gefitinib and erlotinib, to bind to the adenosine triphosphate (ATP)-binding pocket of EGFR, which reduces the EGFR-TKI-mediated inhibition of downstream signaling.

Osimertinib is a novel oral, potent, and selective thirdgeneration irreversible inhibitor of both EGFRm(+) sensitizing and T790M resistance mutants that spares wildtype EGFR. This mono-anilino-pyrimidine compound is structurally distinct from other third-generation EGFR TKIs and offers a pharmacologically differentiated profile from earlier generation EGFR TKIs. Preclinically, the drug potently inhibits signaling pathways and cellular growth 
in both EGFRm(+) and EGFRm(+)/T790M(+) mutant cell lines in vitro, with lower activity against wild-type EGFR lines, translating into profound and sustained tumor regression in EGFR-mutant tumor xenograft and transgenic models (3).

As third-generation EGFR-TKIs selectively act on the EGFR-TKI-sensitive mutations and secondary T790resistant mutation, osimertinib has shown excellent safety and effectiveness in early clinical trials (AURA1 and AURA2) (4,5). Compared with the platinum-containing regimen, the efficacy of osimertinib in the first generation of EGFR-TKI-resistant NSCLC patients was determined by prospective and multicenter clinical trials of AURA3 (2). In this study, the efficacy analysis of osimertinib yielded an ORR of $63.3 \%$, a DCR of $93.3 \%$, an mPFS of

Table 4 The adverse effects of patients with advanced lung adenocarcinoma treated with osimertinib

\begin{tabular}{|c|c|c|c|}
\hline \multirow{2}{*}{ Adverse event } & \multicolumn{2}{|c|}{ Grade } & \multirow{2}{*}{$\%$} \\
\hline & $1-2$ & $3-4$ & \\
\hline Diarrhea & 23 & 3 & 28.9 \\
\hline Rash & 20 & 2 & 24.4 \\
\hline Xerosis cutis & 16 & 0 & 17.8 \\
\hline Onychia lateralis & 12 & 0 & 13.3 \\
\hline Nausea & 10 & 0 & 11.1 \\
\hline Poor appetite & 10 & 0 & 11.1 \\
\hline Constipation & 8 & 0 & 8.9 \\
\hline Anemia & 5 & 0 & 5.6 \\
\hline Thrombocytopenia & 2 & 1 & 3.3 \\
\hline Heart failure & 0 & 1 & 1.1 \\
\hline Dyscrasia & 1 & 0 & 1.1 \\
\hline
\end{tabular}

10.41 months, and an mOS of 31.37 months. Compared with the previous studies $(2,6)$, ORR, DCR, PFS, and OS were comparable. In terms of safety, osimertinib performed well, adverse events were slightly controllable, and the survival period was better.

The FLAURA trial proved that, compared with standard first-line treatment, osimertinib has brought greater benefits to NSCLC patients, especially for the Asian population and patients with brain metastasis (7). Further subgroup analysis of the brain metastasis subgroup found 15 patients with asymptomatic brain metastasis and 6 patients with symptomatic brain metastasis receiving whole brain radiotherapy, with the symptoms and brain lesions being effectively controlled after oral administration of osimertinib. Twenty-one patients with brain metastasis had an ORR of $71.4 \%$, a DCR of $95.2 \%$, and mPFS of 10.21 months, and an mOS of 29.57 months. It was also found that osimertinib could achieve better efficacy and better survival in T790M-positive advanced lung adenocarcinoma patients with brain metastasis.

In $\mathrm{Wu}$ et al.'s study (8), according to the data of the randomized phase III AURA3 trial, the ORR was $40 \%$ (30/75; 95\% CI, 29-52\%) and 17\% (7/41; 95\% CI, 7-32\%) in patients with measurable and/or non-measurable central nervous system (CNS) lesions, respectively $(\mathrm{P}=0.014)$. In patients with measurable and non-measurable CNS lesions, the median CNS remission duration was 8.9 months $(95 \%$ CI, 4.3 months to not calculable) and 5.7 months (95\% CI, 4.4-5.7 months) in the osimertinib group respectively, and 5.6 months $(\mathrm{P}=0.004)$ in the pemetrexed group. Although the number of cases in our study was relatively small, it was still strongly suggested that in the T790M-positive advanced NSCLC patients, the efficacy of osimertinib in the treatment of central nervous system metastasis is relatively accurate.

Table 5 Diagnostic biospecimen type and testing methods for determining T790M mutation status

\begin{tabular}{|c|c|c|c|c|c|}
\hline Biospecimen type & \multicolumn{3}{|c|}{ PCR } & NGS sequencing & Unknown \\
\hline Tissue $(n=11)$ & 2 & 0 & 0 & 10 & - \\
\hline Blood $(n=55)$ & 2 & 50 & 2 & 1 & - \\
\hline Tissue and blood $(n=4)$ & 1 & 4 & 2 & 1 & - \\
\hline Total $(n=90)$ & 5 & 54 & 4 & 12 & - \\
\hline
\end{tabular}

PCR, polymerase chain reaction; ARMS, amplification refractory mutation system; NGS, next-generation sequencing. 
In the subgroup analysis of different sensitive mutations, the ORR and DCR of the 19 del mutation patients were $71.2 \%$ and $96.6 \%$ respectively. The ORR and DCR of patients with another common sensitive mutation, 21 L858R, were $61.1 \%$ and $94.4 \%$ respectively. The effective rates of both were higher than those of unknown and rare mutation patients $(30.8 \%$ and $76.9 \%)$. The difference was statistically significant $(\mathrm{P}=0.020$ and 0.049$)$, but there was no significant difference in $\mathrm{mPFS}$ and $\mathrm{mOS}(\mathrm{P}=0.225$ and $\mathrm{P}=0.941$ ).

The ORR of the T790M mutation-positive patients was $70.3 \%$, while that of the $T 790 M$ mutation-negative or undetected patients was $46.2 \%$. The difference was statistically significant $(\mathrm{P}=0.031)$. The $\mathrm{mPFS}$ of the T790M mutation-positive patients was 12.30 months, while that of the T790M mutation-negative or undetected patients was 6.57 months $(\mathrm{P}=0.004)$. Further analysis found that the T790M mutation was negative in 6 patients. A test for T790M was not carried out in another 20 patients. The specific reasons for this are as follows: (I) the patients were unable to tolerate the traumatic test (the patients were older, the general situation was poor, and there were greater concerns about the trauma of the test); (II) the test cost too high (the cost of peripheral blood test was higher, and medical insurance could not be paid for); (III) the patients and their families refused testing (when there were initial EGFR gene mutation detection results, redetection was considered pointless).

The most common adverse events in this study were diarrhea (28.9\%), rash (24.4\%), dry skin (17.8\%), and paronychia $(13.3 \%)$, which is similar to the AURA3 study. However, in our study, two patients stopped taking osimertinib because of grade 3 thrombocytopenia and heart failure. The specific reason and mechanism for this occurrence needs to be further studied.

Lee et al. (9) found that amplification of the MET gene, amplification of HER2, overexpression of $H G F$, transformation of NSCLC into SCLC and epithelial mesenchymal transformation (EMT) were also important factors of EGFR-TKI acquired resistance. Some secondary mutations of EGFR were also related to the acquired resistance to EGFR-TKI (including the third-generation drug, osimertinib). Our study also suggests that after the first generation of EGFR-TKI resistance, tissue or liquid sample testing (NGS) should be done, on the basis of which heterogeneity should be found and which overall treatment strategy should be implemented.

In summary, for advanced lung adenocarcinoma patients (including those with brain metastasis) with EGFR-T790M mutation-positive after first-line EGFR-TKI treatment, osimertinib can achieve a positive effect and have a low incidence of adverse reactions.

\section{Acknowledgments}

Funding: This work was supported by Basic research cultivation project of Shanghai Chest Hospital (2019YNJCM14), China.

\section{Footnote}

Reporting Checklist: The authors have completed the STROBE reporting checklist. Available at http://dx.doi. org/10.21037/tlcr-20-685

Data Sharing Statement: Available at http://dx.doi. org/10.21037/tlcr-20-685

Conflicts of Interest: All authors have completed the ICMJE uniform disclosure form (available at http://dx.doi. org/10.21037/tlcr-20-685). The authors have no conflicts of interest to declare.

Ethical Statement: The authors are accountable for all aspects of the work in ensuring that questions related to the accuracy or integrity of any part of the work are appropriately investigated and resolved. The study was conducted in accordance with the Declaration of Helsinki (as revised in 2013). Because of the retrospective nature of the research, the requirement for ethical approval and informed consent was waived.

Open Access Statement: This is an Open Access article distributed in accordance with the Creative Commons Attribution-NonCommercial-NoDerivs 4.0 International License (CC BY-NC-ND 4.0), which permits the noncommercial replication and distribution of the article with the strict proviso that no changes or edits are made and the original work is properly cited (including links to both the formal publication through the relevant DOI and the license). See: https://creativecommons.org/licenses/by-nc-nd/4.0/.

\section{References}

1. Bray F, Ferlay J, Soerjomataram I, et al. Global cancer statistics 2018: GLOBOCAN estimates of incidence and 
mortality worldwide for 36 cancers in 185 countries. CA Cancer J Clin 2018;68:394-424.

2. Ricciuti B, Chiari R. First line osimertinib for the treatment of patients with advanced EGFR-mutant NSCLC. Transl Lung Cancer Res 2018;7:S127-30.

3. Cross DA, Ashton SE, Ghiorghiu S, et al. AZD9291, an irreversible EGFR TKI, overcomes T790M-mediated resistance to EGFR inhibitors in lung cancer. Cancer Discov 2014;4:1046-61.

4. Jänne PA, Yang JC, Kim DW, et al. AZD9291 in EGFR inhibitor-resistant non-small-cell lung cancer. N Engl J Med 2015;372:1689-99.

5. Yang J, Ramalingam SS, Jänne PA, et al. LBA2_ PR: Osimertinib (AZD9291) in pretreated pts with T790M-positive advanced NSCLC: Updated phase 1 (P1) and pooled phase 2 (P2) results. J Thorac Oncol

Cite this article as: Gao Z, Wang W, Gu A, Lu J, Huang A, Xiong L, Han B, Jiang L, Shi C. Analysis of the clinical effect of osimertinib on 90 cases with advanced lung adenocarcinoma. Transl Lung Cancer Res 2020;9(4):1464-1471. doi: 10.21037/tlcr20-685
2016;11:S152-S153.

6. Soria JC, Ohe Y, Vansteenkiste J, et al. Osimertinib in Untreated EGFR-Mutated Advanced Non-Small-Cell Lung Cancer. N Engl J Med 2018;378:113-25.

7. First-Line Osimertinib Beneficial in Advanced NSCLC. Cancer Discov 2018;8:OF1.

8. Wu YL, Ahn MJ, Garassino MC, et al. CNS Efficacy of Osimertinib in Patients With T790M-Positive Advanced Non-Small-Cell Lung Cancer: Data From a Randomized Phase III Trial (AURA3). J Clin Oncol 2018;36:2702-9.

9. Lee CK, Kim S, Lee JS, et al. Next-generation sequencing reveals novel resistance mechanisms and molecular heterogeneity in EGFR-mutant non-small cell lung cancer with acquired resistance to EGFR-TKIs. Lung Cancer 2017;113:106-14. 